\title{
Evaluation of Tuberculin Skin Test Results among Students of the University of Medical Sciences and Technology, Sudan
}

\author{
Ahmed H. Babeker ${ }^{1}$, Haitham M. El Bingawi ${ }^{2}$ \\ ${ }^{I}$ (Finalist medical student, Faculty of Medicine, University of Medical Science and Technology) \\ ${ }^{2}$ (Assistant professor of Medicine and Tropical Diseases, Department of Medicine, University of Medical \\ Sciences and Technology)
}

\begin{abstract}
Introduction: Tuberculosis is an endemic infectious disease in Sudan and other developing countries. Healthcare workers, including students, are at a great risk of exposure and possible infection. This study is conducted to determine the pattern and prevalence of positive tuberculin skin test (TST) among students of the University of Medical Sciences and Technology during distinct periods of their training, and to study the influencing factors for positive results.

Design and Setting: A cross-sectional study conducted among 100 students in the period between October 2010 to March 2011 using questionnaires, and administration of the Tuberculin Skin Test (TST) by a licensed professional. Students were divided into two groups, high risk group (medical students), and low risk group (non-medical students) according to their level of exposure. Demographics, BCG vaccination, potential exposure to TB patients and symptomatology were obtained and analyzed.

Results: The overall prevalence of positive TST was 19\%, $2 \%$ were strongly positive, $17 \%$ being moderately positive. The high risk group exhibited a significantly higher rate of positive TST (26\%). Positive TST was found to correlate significantly with contact with TB patients $(P$ 0.08). No significant correlation was found between positive TST and gender $(P 0.834)$, country of residence $(P 0.242)$, vaccination $(P 0.094)$, presence of BCG scar $(P$ 0.793), drinking unpasteurized milk ( $p$ 0.647), immunosuppressive therapy $(P$ 0.674) and crowding $(P$ 0.872 ).

Conclusion: The TST positivity rate was high among our students; it correlates with in-hospital training stage. More attention should be paid to prevention strategies in medical students prior to and during their clinical training.
\end{abstract}

\section{Introduction}

In Sudan, communicable diseases are the leading cause of morbidity and mortality with high vulnerability to outbreaks ${ }^{(1,2, \text { and } 3)}$. Sudan, occupies the third place in the Eastern Mediterranean region in relation to the incidence of tuberculosis, exceeded only by Pakistan and Afghanistan ${ }^{(3,4)}$. It has a prevalence of 209 cases per 100,000 of the population and 50,000 incident cases during $2009{ }^{(5)}$.

Health care workers, particularly those with high level of patient exposure are at increased risk of latent tuberculosis, ${ }^{(6, \text { and } 7)}$. This applies to students in their senior years of clinical training, whose prevalence is higher than the general population ${ }^{(7,8)}$.

At the University of Medical Sciences and Technology, medical students receive their training at different public hospitals, but mainly the Academy Charity Hospital. Being a charity hospital, it cares mostly for the less fortunate of the population who are more prone to be carrying tuberculosis.

Although, an exact figure cannot be named, it is a common knowledge that TB burden at the university teaching hospital is high. Part of tuberculosis infection control programmes in healthcare settings includes evaluation of latent tuberculosis infection ${ }^{(9)}$.

Since the incidence of positive tuberculin skin tests (TSTs) among medical students at the university of Medical Sciences and Technology who may have been infected with Mycobacterium tuberculosis has never been assessed, this cross-sectional study, using TSTs, was conducted.

\section{Material And Method}

A cross-sectional study carried out between October 2010 and March 2011 to estimate the prevalence of positive results of TST and to examine the possible risk factors for being TST positive among students of the University of Medical Sciences and Technology (UMST). UMST, is a private, non-profit making University, located in Khartoum, Sudan, it includes 11 faculties. Students at UMST come from families whose incomes are well above the national average; therefore, it is assumed that the prevalence of latent tuberculosis (TB) or active TB would be low among them than in the general population. At their basic phase of training, medical students at the UMST do not participate in patient examinations or treatment. Contact with patients increases progressively at their clinical training phase. 
A total of 100 students were recruited. 50 were chosen from the high risk group $\left(5^{\text {th }}\right.$ and $4^{\text {th }}$ year medical and Dentistry students) and 50 from the low risk group ( $2^{\text {nd }}$ year Business studies students). The high risk group was defined as a group of students with significant occupational exposure to TB, with increased risk of developing a positive TST. The low risk group was defined as a group of students with no significant occupational exposure to TB, with expected negative TST.

The participation in this study was optional which made the selection bias unavoidable. All selected participants were fully educated about TST by a small talk given prior to the test and by pamphlets distributed among them. The pamphlets outlined the aim, rationale and method of the TST administration and highlighted the possible adverse reactions. The participants were asked to sign a consent form that attached to the questionnaire. Permission was taken from the UMST administration to carry out the research on the premises. BCG vaccination was confirmed by verifying the presence of a vaccination scar over the right deltoid insertion. A disposable needles and 1- $\mathrm{ml}$ syringes were used. A $0.1-\mathrm{ml}$ dose purified protein derivative (PPD) was then administered at the flexor aspect of the left forearm (right arm if the candidate is left handed) by a licensed professional. Induration, not erythema, was measured after 72 hours. An induration above $10 \mathrm{~mm}$ was noted as positive and that of $20 \mathrm{~mm}$ as strongly positive.

Students on immunosuppression therapy were considered positive at a $5 \mathrm{~mm}$ level of induration. Students who present positive TSTs were referred to physician for further assessment. Data was collected by a questionnaire and a master sheet was preformed, the data was then processed and analyzed using Statistical Package for Social Sciences version 16 (SPSS-16). Testing of difference between proportions was conducted using the Chi-Square test, with a value corresponding to $\mathrm{p}<0.05$ for significance unless otherwise stated.

\section{Result}

Of the 100 students examined, 59 were male and 41 were female. Their ages spanned from 16-24, no students was lost during the follow up. 19 (19\%) showed positive TST making the overall prevalence of positive TST 19\%, 2\% of which being strongly positive (Figure 1)

Figure 1: Distribution of study sample according to their results of Tuberculin Skin Test

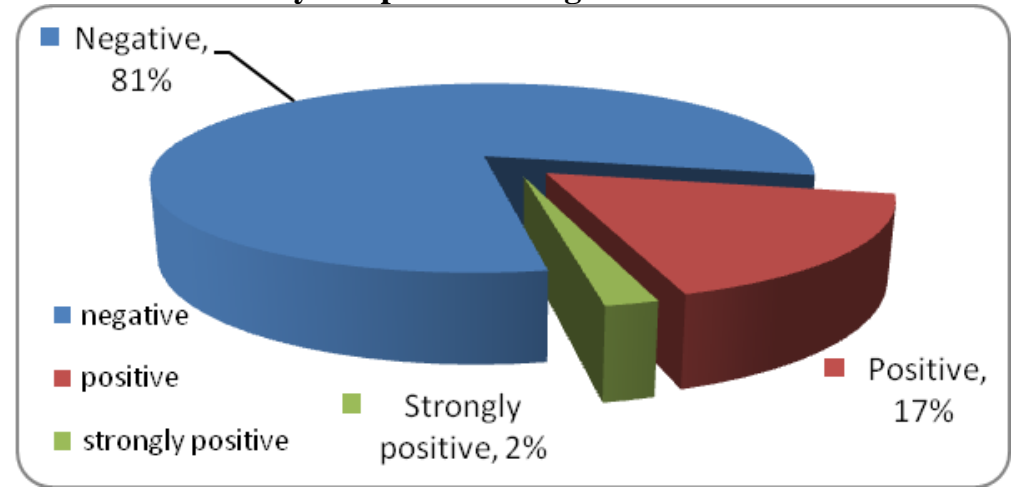

Within the high risk group students, 35 (70\%) were males, 26 (52\%) had lived in developed countries and $24(48 \%)$ in developing countries, 50 (100\%) had close contact with a known tuberculosis patient, $7(14 \%)$ complained of cough, $45(90 \%)$ were previously vaccinated, of which $35(78 \%)$ had a scar present. $37(74 \%)$ had currently a negative TST, $12(24 \%)$ had a positive TST and $1(2 \%)$ had a strongly positive TST. The overall prevalence of positive TST among this group was 26\% (Table 1)

Within the low risk group, there were equal distribution of gender, 24 (48\%) lived in developed countries, $26(52 \%)$ in developing countries, 7 (14\%) had close contact with a known TB patients, 1 (12\%) complained of cough, $1(2 \%)$ had previous TB infection, 29(58\%) were vaccinated, of which $18(62 \%)$ had a scar present. $44(88 \%)$ had currently a negative TST, $5(10 \%)$ had a positive TST and $1(2 \%)$ had a strongly positive TST. The overall prevalence of positive TST among this group was $12 \%$ (Table 1)

All together, 18 students had undergone a previous TST. 14 (78\%) from the high risk group, of which $9(64 \%)$ had a negative result, $4(29 \%)$ had a positive result and $1(7 \%)$ had a strongly positive result. 4 out of 18 (22\%) from the low risk group had undergone a previous TST and they all had a negative result (Table 1) 
Table 1: Characteristics of high risk group and low risk group students participating in the study

\begin{tabular}{|c|c|c|c|c|c|c|c|}
\hline \multicolumn{2}{|c|}{ Characteristics } & \multicolumn{2}{|l|}{$\begin{array}{l}\text { HRG } \\
\text { (number/total) }\end{array}$} & $\%$ & \multicolumn{2}{|l|}{$\begin{array}{l}\mathrm{LRG} \\
(\mathrm{n}=50)\end{array}$} & $\%$ \\
\hline \multirow{2}{*}{\multicolumn{2}{|c|}{ Gender }} & \multicolumn{2}{|l|}{ M 35/50 } & $70 \%$ & \multicolumn{2}{|l|}{$24 / 50$} & $48 \%$ \\
\hline & & \multicolumn{2}{|l|}{ F $26 / 50$} & $52 \%$ & \multicolumn{2}{|l|}{$26 / 50$} & $52 \%$ \\
\hline \multirow{2}{*}{$\begin{array}{l}\text { Resid } \\
\text { ence }\end{array}$} & Living in developed country & \multicolumn{2}{|l|}{$26 / 50$} & $52 \%$ & \multicolumn{2}{|l|}{$24 / 50$} & $48 \%$ \\
\hline & Living in developing country & $24 / 50$ & & $48 \%$ & $26 / 50$ & & $52 \%$ \\
\hline \multicolumn{2}{|c|}{ Contact with TB patients } & \multicolumn{2}{|l|}{$50 / 50$} & $100 \%$ & \multicolumn{2}{|l|}{$7 / 50$} & $14 \%$ \\
\hline \multicolumn{2}{|c|}{ Cough symptom } & \multicolumn{2}{|l|}{$7 / 50$} & $14 \%$ & \multicolumn{2}{|l|}{$1 / 50$} & $12 \%$ \\
\hline \multirow{2}{*}{\multicolumn{2}{|c|}{ History of vaccination }} & \multicolumn{2}{|l|}{$45 / 50$} & $90 \%$ & \multicolumn{2}{|l|}{$29 / 50$} & $58 \%$ \\
\hline & & Presence of BCG scares & $35 / 45$ & $78 \%$ & $\begin{array}{l}\text { Presence of } \\
\text { BCG scares }\end{array}$ & $18 / 29$ & $62 \%$ \\
\hline \multicolumn{2}{|c|}{ Previous TB infection } & \multicolumn{2}{|l|}{-} & - & \multicolumn{2}{|l|}{$1 / 50$} & $12 \%$ \\
\hline \multirow{4}{*}{\multicolumn{2}{|c|}{ Previous TST }} & \multicolumn{2}{|l|}{$14 / 18$} & $78 \%$ & \multicolumn{2}{|l|}{$4 / 18$} & $29 \%$ \\
\hline & & \multicolumn{2}{|l|}{ Negative result $9 / 14$} & $64 \%$ & \multicolumn{2}{|l|}{ Negative $4 / 4$} & $100 \%$ \\
\hline & & \multicolumn{2}{|l|}{ Positive result $4 / 14$} & $29 \%$ & \multicolumn{2}{|l|}{-} & - \\
\hline & & \multicolumn{2}{|l|}{ Strongly positive $1 / 14$} & $7 \%$ & \multicolumn{2}{|l|}{-} & - \\
\hline \multirow{3}{*}{\multicolumn{2}{|c|}{ Current TST result }} & \multicolumn{2}{|l|}{ Negative $37 / 50$} & $74 \%$ & \multicolumn{2}{|l|}{$44 / 50$} & $88 \%$ \\
\hline & & Positive $12 / 50$ & & $24 \%$ & $5 / 50$ & & $10 \%$ \\
\hline & & Strongly positive $1 / 50$ & & $2 \%$ & $1 / 50$ & & $2 \%$ \\
\hline Ingesti & of unpasteurised milk & $8 / 18$ & & $44 \%$ & $10 / 18$ & & $56 \%$ \\
\hline$\overline{\text { On Iml }}$ & unosuppressive therapy & - & & - & $2 /$ & & \\
\hline Prevale & ce of positive TST & $13 / 50$ & & $26 \%$ & $6 / 50$ & & $12 \%$ \\
\hline
\end{tabular}

HRG: High Risk Group; LRG: Low Risk Group; TST: Tuberculin Skin Test; TB: tuberculosis; BCG: Bacillus Calmette-Guérin.

18 students used to drink unpasteurized milk, 8 (44\%) were from the high risk group and $10(56 \%)$ were from the low risk group. Overall, 2 participants from the low risk group were found to be on immunosuppressive therapy and both had a negative TST

Positive TST was found to correlate significantly with contact with TB patients (P 0.08). No significant correlation was found between positive TST and gender ( $p 0.834)$, country of residence ( $p 0.242)$, vaccination ( $p$ 0.094), presence of BCG scar ( $\mathrm{p}$ 0.793), drinking unpasteurized milk ( $\mathrm{p}$ 0.647), immunosuppressive therapy ( $\mathrm{p}$ 0.674), crowding (p 0.872) (Table 2)

Table 2: Analysis of factors correlated with positive Tuberculin Skin Test among the study population.

\begin{tabular}{|c|c|c|c|c|c|c|}
\hline \multicolumn{2}{|l|}{ Characteristics } & \multicolumn{2}{|c|}{+ TST } & \multicolumn{2}{|c|}{-TST } & \multirow[t]{2}{*}{ P Value } \\
\hline & & $\mathrm{N}$ & $\%$ & $\mathrm{~N}$ & $\%$ & \\
\hline Contact with TB patient & & $13 / 57$ & $22.8 \%$ & $42 / 57$ & $(73.7 \%)$ & 0.08 \\
\hline \multirow{2}{*}{\multicolumn{2}{|c|}{ gender }} & $\begin{array}{l}M \\
14 / 19\end{array}$ & $73.7 \%$ & 45 & $(76.3 \%)$ & \multirow[t]{2}{*}{0.8} \\
\hline & & F 5/19 & $26.3 \%$ & 36 & $(87.8 \%)$ & \\
\hline \multicolumn{2}{|l|}{ Vaccination } & $16 / 74$ & $21.6 \%$ & $57 / 74$ & $(77 \%)$ & 0.09 \\
\hline \multicolumn{2}{|l|}{ BCG scar } & $11 / 53$ & $20.8 \%$ & $41 / 53$ & $(77.4 \%)$ & 0.793 \\
\hline \multirow{2}{*}{ Residence } & Developing countries & $12 / 50$ & $24 \%$ & $38 / 50$ & $(76 \%)$ & \multirow[b]{2}{*}{0.2} \\
\hline & Developed countries & $7 / 50$ & $14 \%$ & 43 & $(86.8 \%$ & \\
\hline Drinking Unpasteurized Milk & & $2 / 50$ & $11 \%$ & 16 & $88.8 \%$ & 0.7 \\
\hline
\end{tabular}

TST: Tuberculin Skin Test; TB: tuberculosis; BCG: Bacillus Calmette-Guérin.

\section{Discussion}

In this study, an attempt was made to determine the risk of infection and to correlate risk factors contributing to a positive Tuberculin Skin Test among the study population. For this purpose, a cross-sectional study was conducted, however, in similar studies it would be more suitable if longitudinal study was done ${ }^{(6)}$. The overall prevalence of positive TST observed in this study was higher than that observed in many other studies, perhaps; it was conducted in a country with higher incidence of pulmonary tuberculosis ${ }^{(3)}$. Furthermore, safety measures that could reduce transmission of infections among students were not appropriately applied in the hospitals where they get their training (...).

Appropriate applications of universal measures may reduce the risk of TB transmission ${ }^{(10,11, \text { and } 12)}$. On the other hand, 37(74\%) students of the high risk group had a negative TST; perhaps, they have never been 
exposed to mycobacterium tuberculosis infection. Therefore, they are at a great risk of contracting the infection, taking in to account the high prevalence of positive TST noticed in this study and the possible low biosafety measures in the hospitals.

The study showed a clear correlation between positive TST and contact with TB patients. This is in keeping with other studies ${ }^{(6.7}$ and 8$)$. Overall, $74 / 100$ students were vaccinated, most likely they were those born in Sudan or the gulf countries where BCG is administered routinely to all neonates as part of the WHO immunization program. Unlike western countries, were only minority of the population is subjected to BCG vaccination ${ }^{(13)}$. No correlation was found between TST conversion and vaccination; this could be explained by the long time period between vaccination (at birth) and the TST(at current study). Studies have shown that the closer the time of vaccination the larger the area of induration.

No association was found between positive TST and number of students per room, this is acceptable as only $1 \%$ having 4 per room, and none had more. This can be attributed to the high socioeconomic status of students attending UMST.

This study showed no association between TST conversion and gender. This is the norm for most studies ${ }^{(6,7)}$. A paper by Teixeira, et al explore different view, in which TST was found to correlate positively with male gender; it was attributed to the behavior patterns, as men are more likely to undertake risky behavior and to ignore recommendations for preventive measures ${ }^{(8)}$.

No association was found between TST conversion and immunosuppressive therapy, even after setting the bar for a positive at lower level of indurations $(5 \mathrm{~mm})$.

\section{Conclusion}

Limitations: It is possible that students who knew that they were TST reactive avoided participating in the study and that those who had been previously exposed to TB patients were more interested in participating.

The study concludes that the tuberculin skin test rate was high (19\%) among our medical and dentistry students in their final years of clinical training. The main associated factor being the high level of unprotected exposure attained at the teaching hospitals and therefore, more attention should be paid to prevention strategies in medical students prior to and during their clinical training

\section{Recommendations:}

1. BCG vaccinations for all those with a negative TST, especially those among the high risk group.

2. Implementation of a TB control program at the university teaching hospitals.

3. Routine TST and BCG vaccine for medical students. This would be a simple yet strong method of eradicating any risks that the medical students may face, this is particularly relevant to UMST since many of the students have been born and raised in western countries and for those who have received the BCG vaccine long time ago.

4. A similar study should be done on a wider scale among the medical student population within Sudan.

[1] World Health Organization. Country Cooperation Strategy for WHO and Sudan (2006). Available at: http://www.who.int/country focus/cooperation strategy/ccs_sdn_en.pdf.

[2] FMOH (Federal Ministry of Health). 5-year Health Sector Strategy: Investing in Health and Achieving the MDGs (2007). Available at: http://www.fmoh.gov.sd/English/index.php?id=2

[3] Sharaf Eldin G S, Fadl-Elmula I, Ali MS, et al. Tuberculosis in Sudan: a study of Mycobacterium tuberculosis strain genotype and susceptibility to anti-tuberculosis drugs BMC Infectious Diseases 2011, 11:219

[4] World Health Organization. TB anywhere is TB everywhere (2007). Available at http://www.emro.who.int/stb/tbday07-kit-tb$\underline{\text { emr.htm }}$

[5] World Health Organisation: Global tuberculosis control: WHO Report 2010. Geneva; 2010.

[6] Soares LCP, Mello FCQ, Kritski AL. Prevalence of tuberculin skin testing among medical students in Campos School of Medicine, Rio de Janeiro. Jornal Brasileiro de Pneumologia 2004;30(4)

[7] Silva VMC, Cunha AJA, Kritski AL. Tuberculin Skin Test Conversion Among Medical Students at a Teaching Hospital in Rio de Janeiro, Brazil. Infection Control and Hospital Epidemiology 2002; 23(10): 591- 594.

[8] Teixeira EG, Menzies D, Comstock GW, et al. Latent Tuberculosis Infection among Undergraduate Medical Students in Rio de Janeiro State, Brazil E. G. Teixeira. International Journal of Tuberculosis and Lung Disease 2005; 9(8): 841-847.

[9] American Thoracic Society/Centers for Disease Control and Prevention/Infectious Diseases Society of America. Controlling tuberculosis in the United States. Am J Respir Crit Care Med 2005;172:1169-1227.

[10] Menzies D, Fanning A, Yuan Lilian, et al. Tuberculosis among Health Care Workers. Engl J Med 1995; 332:92-98

[11] Maloney SA, Pearson MT, Del Castillo JF, et al. Efficacy of control measures in preventing nosocomial transmission of multidrugresistant tuberculosis to patients and health care workers. Centers for Disease Control and Prevention, Atlanta, GA. Annals of Internal Medicine[1995, 122(2):90-95]

[12] Harries A D, Maher D, Nunn P. Practical and affordable measures for the protection of health care workers from tuberculosis in low-income countries. Bull World Health Organ. 1997; 75(5): 477-489.

[13] Meier T, Eulenbruch HP, Wrighton-Smith P, et al. Sensitivity of a new commercial enzyme-linked immunospot assay (T SPOTTB) for diagnosis of tuberculosis in clinical practice. European Journal of Clinical Microbiology \& Infectious Diseases 2005; 24(8): 529-36. 